\title{
TRAP1 role in endoplasmic reticulum stress protection favors resistance to anthracyclins in breast carcinoma cells
}

\author{
LORENZA SISINNI $^{1}$, FRANCESCA MADDALENA ${ }^{1}$, GIACOMO LETTINI $^{1}$, VALENTINA CONDELLI ${ }^{1}$, \\ DANILO SWANN MATASSA ${ }^{2}$, FRANCA ESPOSITO ${ }^{2}$ and MATTEO LANDRISCINA ${ }^{3}$ \\ ${ }^{1}$ Laboratory of Pre-Clinical and Translational Research, IRCCS, Referral Cancer Center of Basilicata, Rionero in Vulture, \\ Potenza; ${ }^{2}$ Department of Molecular Medicine and Medical Biotechnologies, University of Naples Federico II, Naples; \\ ${ }^{3}$ Clinical Oncology Unit, Department of Medical and Surgical Sciences, University of Foggia, Foggia, Italy
}

Received September 5, 2013; Accepted October 21, 2013

DOI: 10.3892/ijo.2013.2199

\begin{abstract}
Adaptation to endoplasmic reticulum (ER) stress through the upregulation of the ER chaperone BiP/Grp78 favors resistance of cancer cells to anthracyclins. We recently demonstrated that the mitochondrial HSP90 chaperone TNF receptor-associated protein 1 (TRAP1) is also localized in the $\mathrm{ER}$, where it is responsible for protection from ER stress and quality control on specific mitochondrial proteins contributing to its anti-apoptotic function and the regulation of the mitochondrial apoptotic pathway. Based on the evidence that Bip/Grp78 and TRAP1 are co-upregulated in about 50\% of human breast carcinomas (BCs), and considering that the expression of TRAP1 is critical in favoring resistant phenotypes to different antitumor agents, we hypothesized that ER-associated TRAP1 is also favoring resistance to anthracyclins. Indeed, anthracyclins induce ER stress in BC cells and cross-resistance between ER stress agents and anthracyclins was observed in bortezomib- and anthracyclin-resistant cells. Several lines of evidence suggest a mechanistic link between the ER-stress protecting function of TRAP1 and resistance to anthracyclins: i) ER stress- and anthracyclin-resistant cell lines
\end{abstract}

Correspondence to: Professor Franca Esposito, Dipartimento di Medicina Molecolare e Biotecnologie Mediche, Università degli Studi di Napoli Federico II, Via S. Pansini 5, I-80131 Napoli, Italy

E-mail: franca.esposito@unina.it

Dr Matteo Landriscina, Dipartimento di Scienze Mediche e Chirurgiche, Università degli Studi di Foggia, Viale Pinto 1, I-71100 Foggia, Italy

E-mail: m.landriscina@unifg.it

Abrreviations: TRAP1, TNF receptor-associated protein 1; HSP, heat shock proteins; KD, knockdown; PERK, PRKR-like endoplasmic reticulum kinase; UPR, unfolded protein response; ER, endoplasmic reticulum; shRNA, short-hairpin RNA; GAPDH, glyceraldehyde-3phosphate dehydrogenase

Key words: TNF receptor-associated protein 1, anthracyclins, apoptosis, endoplasmic reticulum stress, drug resistance are characterized by the upregulation of TRAP1; ii) TRAP1 silencing in both drug-resistant cell models restored the sensitivity to bortezomib and anthracyclins; iii) the transfection of a TRAP1 deletion mutant, whose localization is restricted to the ER, in TRAP1 KD cells protected from apoptosis induced by anthracyclins; iv) the disruption of the ER-associated TRAP1/TBP7 pathway by a TBP7 dominant negative deletion mutant re-established drug sensitivity in drug-resistant cells. This process is likely mediated by the ability of TRAP1 to modulate the PERK pathway as TRAP1 KD cells failed to induce the phosphorylation of PERK in response to anthracyclins. Moreover, the downregulation of TRAP1 in combination with ER stress agents produced high cytotoxic effects in BC cells. These results suggest that ER-associated TRAP1 plays a role in protecting tumor cells against DNA damaging agents by modulating the PERK pathway.

\section{Introduction}

Protein folding and degradation pathways are strictly regulated in normal cells to avoid accumulation of misfolded proteins and maintain protein homeostasis. When the accumulation of misfolded proteins exceeds degradation, as often occurs in damaged or aging cells or in cells exposed to chemical agents that perturb protein folding, the unfolded protein response (UPR) is elicited to re-establish protein homeostasis and prevent apoptotic cell death (1). However, the persistence of endoplasmic reticulum (ER) stress conditions can switch on an apoptotic program, which finally results in cell elimination $(2,3)$. The development and the progression of cancer is associated to the dysregulation of this process and the persistent activation of the adaptive ER stress response. Indeed, tumor cells are characterized by increased rates of protein synthesis to fulfill the high metabolic demand of accelerated proliferation and, thus, are chronically exposed to ER stress conditions $(4,5)$. In such a scenario, chronic ER stress adaptive responses are involved in tumor progression, adaptation to unfavorable environmental conditions and resistance to cytotoxic agents and, therefore, are regarded as novel targets for the development of new cancer therapeutics (6). Furthermore, recent studies suggest that the ER stress response, besides being responsible for the resistance to pharmacological agents that 
perturb protein homeostasis (7), may favor resistance to DNA damaging agents (8).

Our group has contributed to the demonstration that the mitochondrial chaperone TRAP1 is involved in protection against ER stress (9-11). Indeed, TRAP1 is an HSP90 homolog, originally described as a protein responsible for the prevention of mitochondrial apoptosis, due to its interaction with HSP90 and cyclofilin D and its regulatory property on mitochondrial transition pore (MTP) (12). As TRAP1 is selectively upregulated in several human malignancies (i.e., colorectal, breast and prostate carcinomas) (13-15), its role in protecting against the cytotoxic activity of antiblastic agents in human malignancies has been widely demonstrated $(13,16,17)$.

More recent evidence described the presence of TRAP1 on the outer side of the ER at the interface with mitochondria, where this chaperone interacts with TBP7, an AAA-ATPase of the 19S proteasomal subunit, and is responsible for the modulation of the ER stress response and the quality control of specific mitochondrial client proteins (9), this resulting in protection against mitochondrial apoptosis (18). Indeed, we recently demonstrated that the ER-stress protecting activity of TRAP1 is crucial for its antiapoptotic function and favors resistance against taxanes in breast carcinoma (BC) cells (18). In this context, we observed that ER-associated TRAP1 modulates the mitochondrial apoptotic pathway by regulating the quality of specific client proteins and, among others, $18 \mathrm{kDa}$ sorcin, a mitochondrial protein involved in the TRAP1 cytoprotective pathway (17), this likely representing a major mechanism responsible for the survival response elicited by ER-associated TRAP1 $(9,18)$.

Based on these premises, we investigated the role of ER-associated TRAP1 in favoring resistance to anthracyclins, cytotoxic agents among the most effective anticancer drugs ever developed $(19,20)$. Indeed, it has been hypothesized that the activation of the UPR in the ER is responsible for resistance to genotoxic agents such as topoisomerase inhibitors and platin derivatives $(8,21)$. High expression of the ER chaperone $\mathrm{BiP} / \mathrm{Grp} 78$ is protective against apoptotic stimuli in cancer cells (22), favors resistance to anti-estrogen therapy and chemotherapeutics in several cancer cell models $(8,21,23)$ and correlates with shorter overall survival in human prostate (24) and lung cancer (22). The cytoprotective activity of ER-associated TRAP1 against anthracyclins was investigated in BC cells since our group recently demonstrated that TRAP1 and $\mathrm{BiP} / \mathrm{Grp} 78$ are co-upregulated in about $50 \%$ of human BCs (18) and considering that anthracyclins are highly effective agents in these malignancies (25). Here, we report that TRAP1 regulation of ER stress response is critical in favoring resistance to anthracyclins and provide evidence that this response involves the modulation of PERK pathway.

\section{Materials and methods}

Cells, plasmids and chemicals. Human MCF7 BC cells were purchased from ATCC (Manassas, VA, USA) and cultured in DMEM containing $10 \%(\mathrm{v} / \mathrm{v})$ fetal bovine serum in standard conditions. Cell line authentication was performed by DNA profiling, according to ATCC product description. Drug-resistant cells were selected as previously reported $(18,26)$. siRNAs of TRAP1 were purchased from Qiagen
(Milan, Italy; cat. nos. SI00115150 for TRAP1 and SI03650318 for negative control), diluted to a final concentration of $20 \mathrm{nmol} / 1$ and transfected according to the manufacturer's protocol by using HiPerFect Transfection Reagent (Qiagen). Constructs encoding for wild-type TRAP1, and $\Delta 1$-59TRAP1-Myc and TBP7-Flag deletion mutants (9) were transiently transfected with Polyfect Transfection reagent (Qiagen). Unless otherwise specified, reagents were purchased from Sigma-Aldrich (Milan, Italy).

Immunoblot analysis and antibodies. Total cell lysates were obtained by homogenization of cell pellets in cold lysis buffer (20 mM Tris, pH 7.5 containing $300 \mathrm{mM}$ sucrose, $60 \mathrm{mM}$ $\mathrm{KCl}, 15 \mathrm{mM} \mathrm{NaCl}, 5 \%$ (v/v) glycerol, $2 \mathrm{mM}$ EDTA, $1 \%$ (v/v) Triton X-100, $1 \mathrm{mM}$ PMSF, $2 \mathrm{mg} / \mathrm{ml}$ aprotinin, $2 \mathrm{mg} / \mathrm{ml}$ leupeptin and $0.2 \%(\mathrm{w} / \mathrm{v})$ deoxycholate) for $1 \mathrm{~min}$ at $4^{\circ} \mathrm{C}$ and further sonication for $30 \mathrm{sec}$ at $4^{\circ} \mathrm{C}$. Immunoblot analysis was performed as previously reported $(26,27)$. Briefly, equal amounts of protein from cell lysates were separated by SDS-PAGE and transferred to a nitrocellulose support (Bio-Rad, Hercules, CA, USA). Specific proteins were detected by using the following antibodies: mouse monoclonal anti-GAPDH (sc-47724, Santa Cruz Biotechnology, Segrate, Italy), mouse monoclonal anti-TRAP1 (sc-13557, Santa Cruz Biotechnology), rabbit polyclonal anti-caspase 12 (SPA-827; StressGen, Milan, Italy), rabbit polyclonal anti-Grp94 (sc-11402, Santa Cruz Biotechnology), rabbit polyclonal anti-phosphoPERK (Thr 981, sc-32577, Santa Cruz Biotechnology), rabbit monoclonal anti-PERK (\#3192, Cell Signaling Technology, Boston, MA, USA), rabbit polyclonal anti-LC3B (\#2775, Cell Signaling Technology), mouse monoclonal anti-BiP/Grp78 (E-4, sc-166490, Santa Cruz Biotechnology), mouse monoclonal anti-uniquitin (P4D1, sc-8017, Santa Cruz Biotechnology), mouse monoclonal anti-Myc (9E10, sc-40, Santa Cruz Biotechnology), and rabbit polyclonal anti-Flag (sc-807, Santa Cruz Biotechnology) antibodies. Proteins were visualized with an ECL detection system (Bio-Rad).

RNA extraction and semiquantitative and Real-time RT-PCR analysis. Total RNA from cell pellets was extracted using the TRIzol Reagent (Invitrogen, San Giuliano Milanese, Italy), For the first strand synthesis of cDNA, $1 \mu \mathrm{g}$ of RNA was used in a $20 \mu \mathrm{l}$ reaction mixture utilizing a Transcriptor First Strand cDNA Synthesis Kit (Roche, Mannheim, Germany). A total of $5 \mu \mathrm{l}$ of cDNA sample were amplified using the LightCycler 480 SYBR-Green I Master (Roche) in an Light Cycler 480 (Roche). The following primers were used: BiP/Grp78, forward 5'-GTGGAATGACCCGTCTGTC-3', reverse 5'-CGTCTTTG GTTGCTTGGC-3' (PCR product $254 \mathrm{bp}$ ); GAPDH forward 5'-AGGCTGAGAACGGGAAGC-3', reverse 5'-CCATGGTG GTGAAGACGC-3' (PCR product $135 \mathrm{bp}$ ). Primers were designed to be intron spanning. Reaction condition were as follows: pre-incubation at $95^{\circ} \mathrm{C}$ for $5 \mathrm{~min}$, followed by 45 cycles of $10 \mathrm{sec}$ at $95^{\circ} \mathrm{C}, 7 \mathrm{sec}$ at $60^{\circ} \mathrm{C}, 10 \mathrm{sec}$ at $72^{\circ} \mathrm{C}$. GAPDH was chosen as an internal control.

Apoptosis assay. Apoptosis was evaluated by cytofluorimetric analysis of Annexin V and 7-amino-actinomycin-D (7-AAD)-positive cells using the fluorescein isothiocyanate 
A

- Viable cells $\quad$ Apoptotic cells

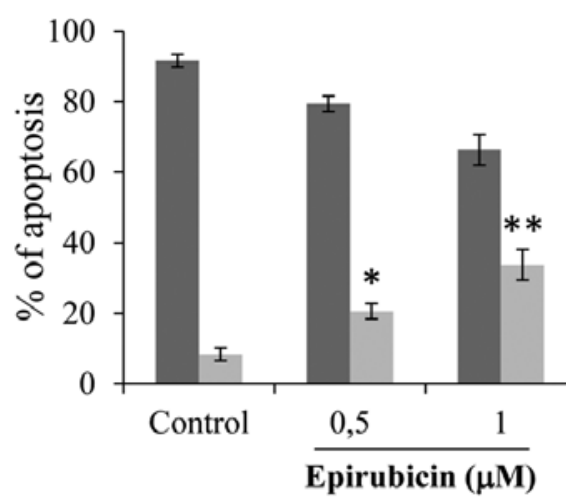

B
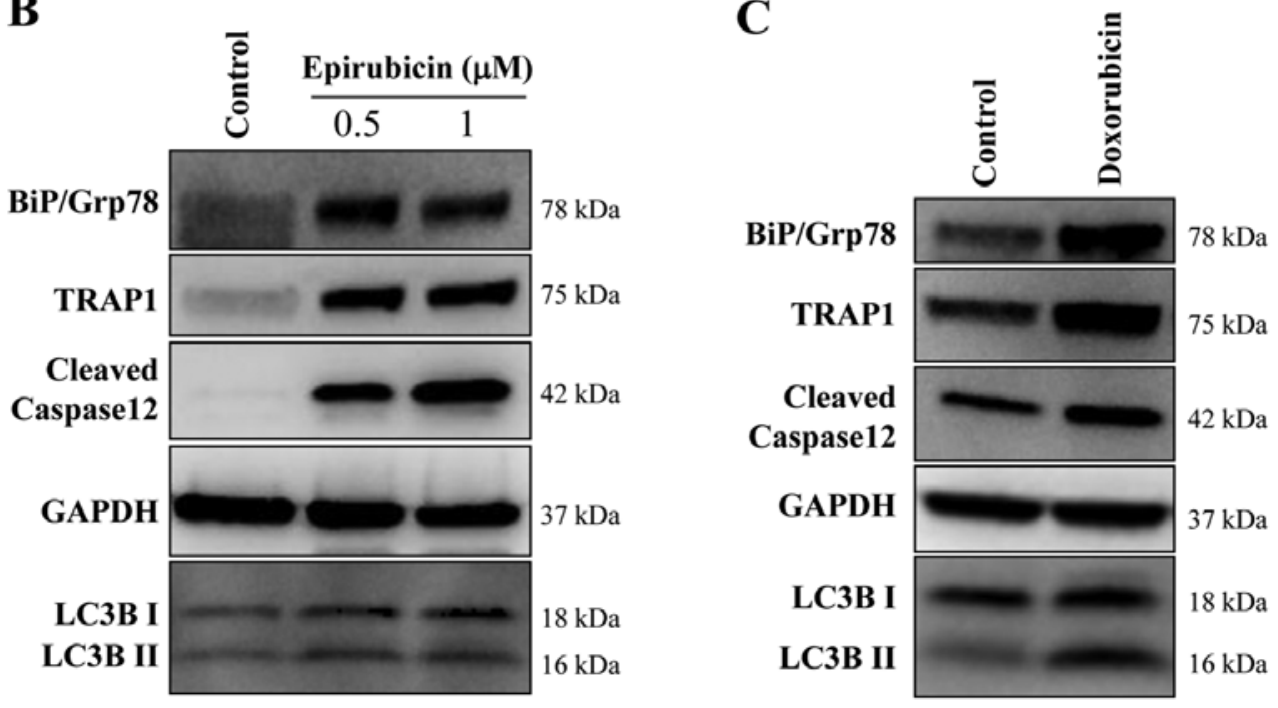

Figure 1. Cytotoxic activity of anthracyclins correlates with ER stress induction. (A) Apoptotic levels in MCF7 cells treated with 0.5 and $1 \mu$ M epirubicin or 1 and $5 \mu \mathrm{M}$ doxorubicin for $24 \mathrm{~h}$. P-values versus the respective untreated control $\left({ }^{*} \mathrm{p}=0.002 ;{ }^{* *} \mathrm{p}<0.0001\right)$. Total cell lysates from MCF7 cells treated with (B) 0.5 and $1 \mu \mathrm{M}$ epirubicin or (C) $5 \mu \mathrm{M}$ doxorubicin for $24 \mathrm{~h}$ were separated by SDS-PAGE and immunoblotted with anti-BiP/Grp78, anti-TRAP1, anti-caspase 12 , anti-GAPDH and anti-LCII/III antibodies.

(FITC)-Annexin V/7-AAD kit (Beckman Coulter, Milan, Italy). Stained cells were analyzed by using the FACSCalibur ${ }^{\mathrm{TM}}$ (Becton-Dickinson). Ten thousand events were collected per sample. Positive staining for Annexin $\mathrm{V}$ as well as double staining for Annexin $\mathrm{V}$ and 7-AAD were interpreted as signs of, respectively, early and late phases of apoptosis, as previously reported (28).

Statistical analysis. The paired Student's t-test was used to establish the statistical significance between different levels of apoptosis or gene expression in controls and treated cells or in transfected cells and related scramble controls. Statistically significant values $(\mathrm{p}<0.05)$ are reported in Fig. legends.

\section{Results}

The ER stress protecting activity of TRAP1 is relevant for resistance of $B C$ cells to anthracyclins. Since anthracyclins have been proposed to induce ER stress conditions and Bip/Grp78 expression (8), we preliminarily confirmed that the exposure of MCF7 cells to cytotoxic concentrations of both epirubicin and doxorubicin (Fig. 1A) elicits an ER UPR characterized by high levels of BiP/Grp78 and the autophagy marker LC3, and the cleavage of caspase 12 (Fig. 1B). Since high expression of BiP/Grp78 and adaptation to ER stress conditions have been suggested to favor resistance to anthracyclins $(8,21,29)$, we questioned whether BC cells adapted to ER stress conditions may be cross-resistant to doxorubicin and epirubicin. To this purpose we used MCF7 cells chronically adapted to toxic concentrations of the proteasome inhibitor, bortezomib, previously selected in our laboratory (18) and characterized by increased $\mathrm{BiP} / \mathrm{Grp} 78$ and Grp94 expression (Fig. 2A). Of note, cross-resistance was observed upon exposure of bortezomib-resistant cells to cytotoxic concentrations of doxorubicin and epirubicin (Fig. 2B). Since i) TRAP1 involvement in ER stress protection was previously described (9); ii) the exposure of MCF7 cells to anthracyclins resulted in the upregulation of TRAP1 (Fig. 1B); and iii) bortezomib-resistant cells exhibited increased TRAP1 levels (Fig. 2A), we further investigated the role of TRAP1 in favoring resistance to anthracyclins. To this aim, TRAP1 was transiently interfered in bortezomib-resistant cells (Fig. 2B, insert), this resulting in re-establishment of sensitivity to epirubicin and doxorubicin and bortezomib (Fig. 2B). 
A

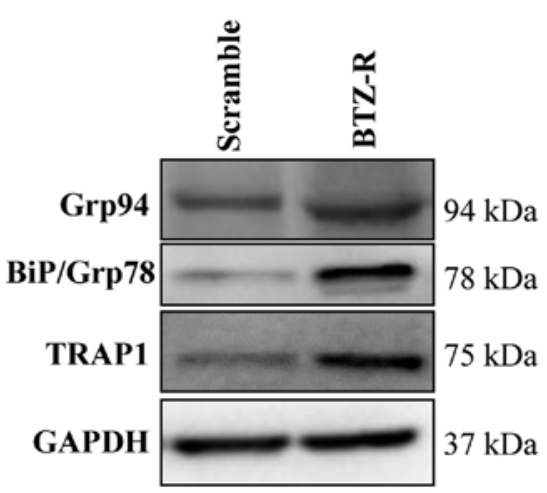

B

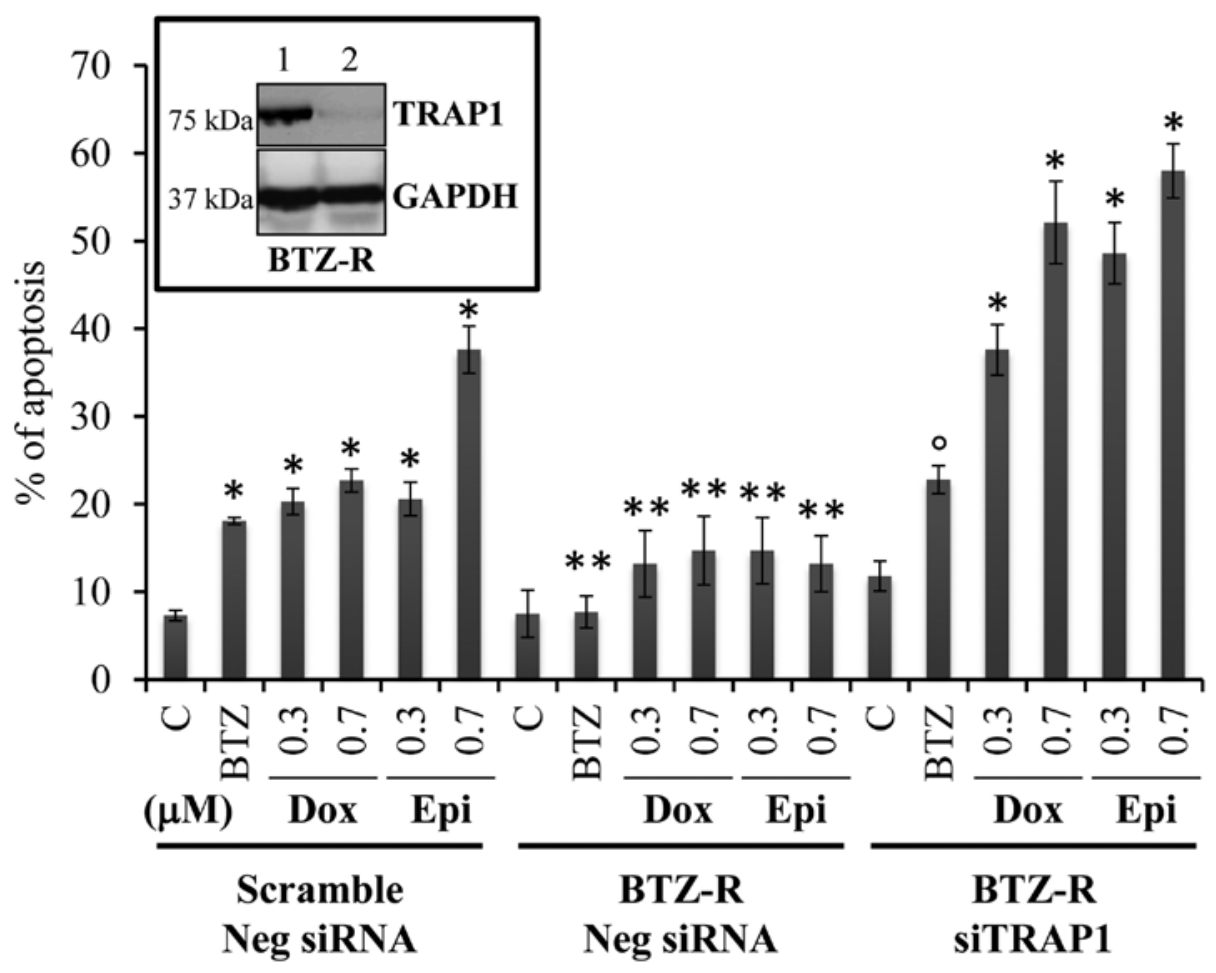

Figure 2. Bortezomib-adapted cells are resistant to anthracyclins. (A) Total cell lysates from scramble and bortezomib-resistant (BTZ-R) MCF7 cells were separated by SDS-PAGE and immunoblotted with anti-Grp94, anti-BiP/Grp78, anti-TRAP1 and anti-GAPDH antibodies. (B) Apoptotic levels in scramble and bortezomibresistant (BTZ-R) MCF7 cells transfected with negative (Neg) or TRAP1 siRNA (siTRAP1) treated with $50 \mathrm{nM}$ BTZ, or 0.5 and $0.7 \mu \mathrm{M}$ doxorubicin (DOX) or epirubicin (Epi) for $24 \mathrm{~h}$. P-values versus the respective untreated control ( ${ }^{*} \mathrm{p}<0.0001 ;{ }^{* *} \mathrm{p}$, not significant; $\left.{ }^{\circ} \mathrm{p}=0.01\right)$. Insert, TRAP1 levels in bortezomib-resistant MCF7 cells upon transfection with (1) negative or (2) TRAP1 siRNA.

To characterize the involvement of TRAP1 in resistance to anthracyclins we generated two further MCF7 cell lines resistant to epirubicin and doxorubicin. Both anthracyclin-resistant cell lines showed increase in total protein ubiquitination and TRAP1 (Fig. 3A), as well as BiP/Grp78 upregulation at protein (Fig. 3A) and mRNA (Fig. 3B) levels. These anthracyclinresistant cell models, besides being resistant to the respective selection agent, showed cross-resistance to the ER stress agent bortezomib (Fig. 3C and D). Consistently with previous results, the downregulation of TRAP1 by siRNA in both anthracyclinresistant cell lines (Fig. 3C and D, inserts) resulted in the re-establishment of the sensitivity to doxorubicin and epirubicin, as well as bortezomib (Fig. 3C and D). These results suggest that adaptation to ER stress and anthracyclins share common mechanisms and that TRAP1 is part of this process.
ER-associated TRAPI is responsible for cytoprotection against anthracyclins. Previous evidence suggests that TRAP1 anti-apoptotic function relies on a dual mechanism: a direct regulation of cyclophillin D folding and MTP opening within mitochondria (12) and a quality control on specific mitochondrial anti-apoptotic client proteins in the ER (18). Thus, we questioned whether this cytoprotective activity of ER-associated TRAP1 is relevant in favoring resistance to anthracyclins. To this purpose we used BC MCF7 cells with low TRAP1 levels (shTRAP1 MCF7 cells, Fig. 4A, insert), transiently transfected with TRAP1 expression vector (Fig. 4A, insert) or the $41-59 \mathrm{TRAP1}$ deletion mutant (Fig. 4B, insert), which lacks the mitochondrial targeting sequence and, thus is unable to enter into mitochondria, while still able to bind TBP7 and protect from ER stress $(9,18)$. We 

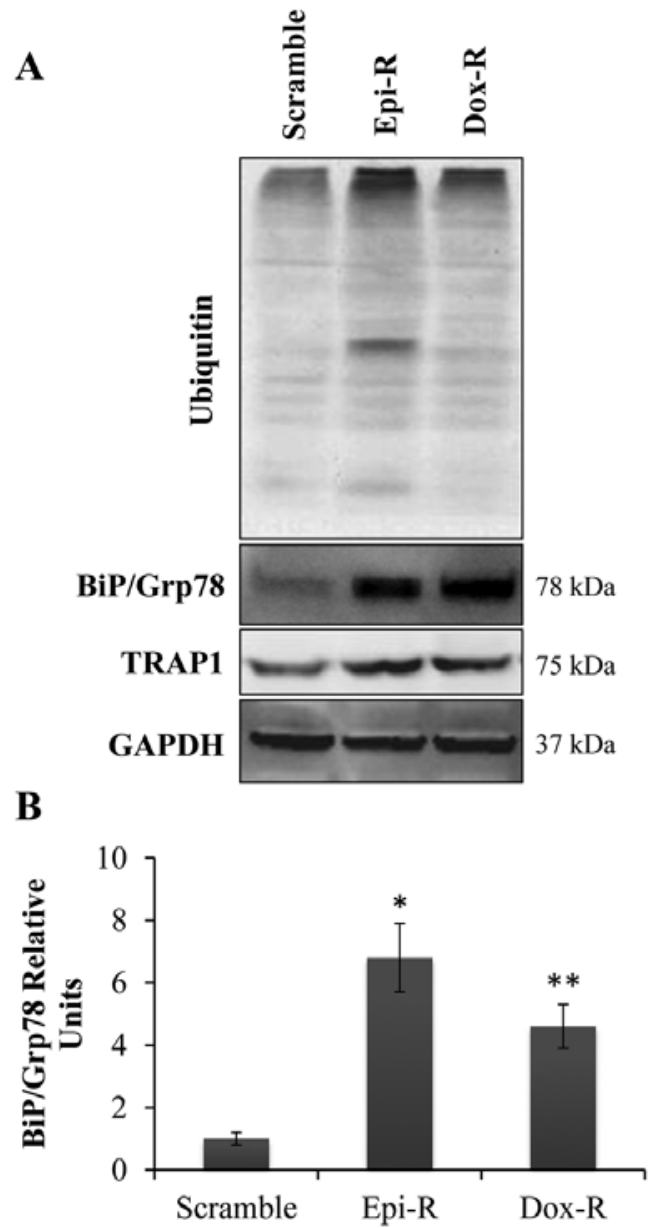
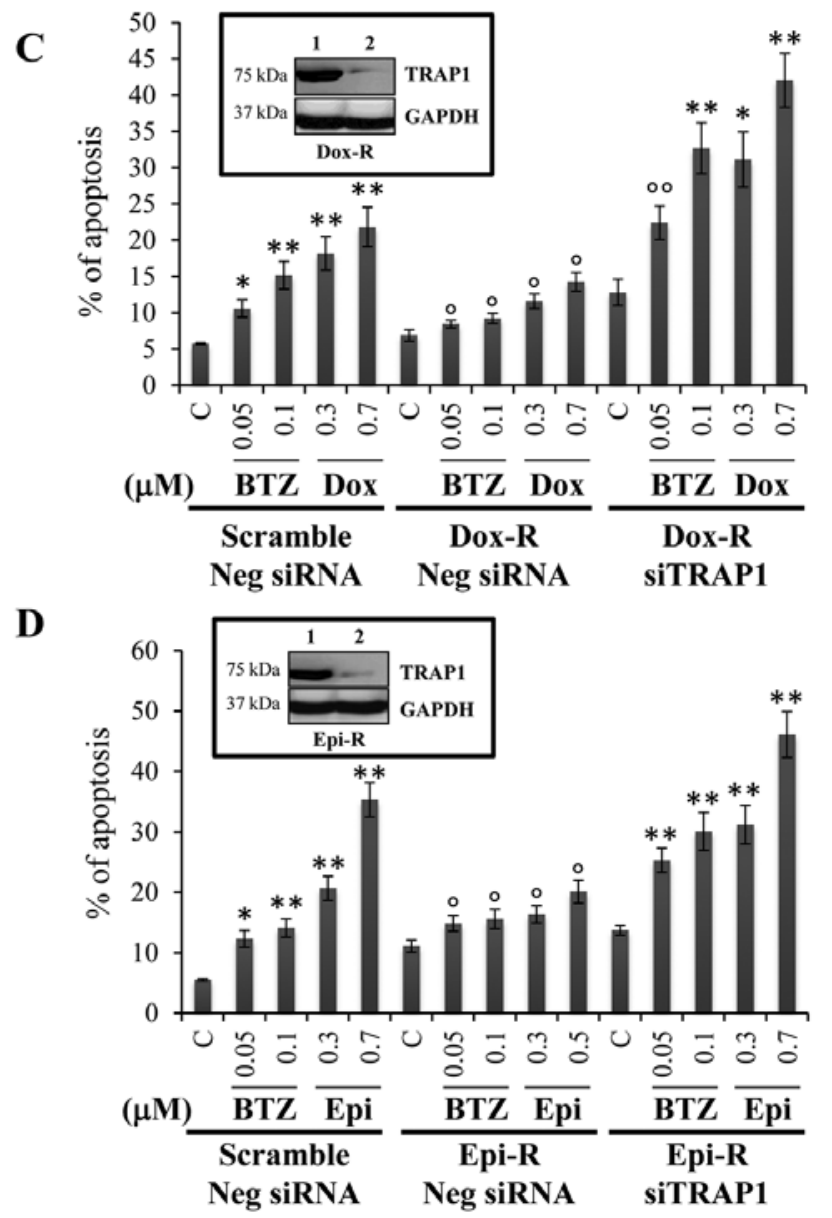

Figure 3. TRAP1 is involved in resistance to anthracyclins. (A) Total cell lysates from scramble, epirubicin-resistant (Epi-R) and doxorubicin-resistant (Dox-R) MCF7 cells were separated by SDS-PAGE and immunoblotted with anti-ubiquitin, anti-BiP/Grp78, anti-TRAP1 and anti-GAPDH antibodies. (B) Bip/Grp78 mRNA levels in scramble, Epi-R and Dox-R MCF7 cells. P-values versus scramble cells ( $\left.{ }^{*} \mathrm{p}<0.0001 ;{ }^{* *} \mathrm{p}=0.001\right)(\mathrm{C})$ Apoptotic levels in scramble and Dox-R MCF7 cells tansfected with negative (Neg) or TRAP1 siRNA (siTRAP1) treated with 50 and $100 \mathrm{nM}$ BTZ, or 0.3 and $0.7 \mu \mathrm{M}$ doxorubicin (Dox) for $24 \mathrm{~h}$. P-values versus the respective untreated control ( $\left(\mathrm{p}=0.002 ;{ }^{* *} \mathrm{p}<0.0001 ;{ }^{\circ} \mathrm{p}\right.$, not significant; $\left.{ }^{\circ} \mathrm{p}=0.005\right)$. (D) Apoptotic levels in scramble and Epi-R MCF7 cells tansfected with negative (Neg) or TRAP1 siRNA (siTRAP1) and treated with 50 and $100 \mathrm{nM}$ BTZ, or 0.3 and $0.5 \mu \mathrm{M}$ epirubicin (Epi) for $24 \mathrm{~h}$. P-values versus the respective untreated control ( ${ }^{*} \mathrm{p}=0.001 ;{ }^{* *} \mathrm{p}<0.0001 ;{ }^{\circ} \mathrm{p}$, not significant). (C and D) Inserts, TRAP1 levels in (C) Dox-R and (D) Epi-R MCF7 cells upon transfection with (1) negative or (2) TRAP1 siRNA.

questioned whether this TRAP1 mutant was able to protect from apoptosis induced by the ER stress agent, bortezomib and/or anthracyclins. Interestingly, we observed a significant cytoprotective activity toward the proteasome inhibitor, as well as doxorubicin and epirubicin due to re-expression of both wild-type TRAP1 (Fig. 4A) and the mitochondrial importdefective TRAP1 mutant (Fig. 4B). In order to further support the cytoprotective function of ER-associated TRAP1/TBP7 pathway we used a TBP7 deletion mutant, which was previously generated by our group, lacking TRAP1 binding site and, characterized for a dominant negative function over the endogenous TBP7 protein function (9). The TBP7 deletion mutant was transfected in scramble MCF7 (Fig. 4C, insert) and in MCF7 cells adapted to docetaxel (Fig. 4C), a cytotoxic which acts by inducing ER stress (30) or in MCF7 cells adapted to anthracyclins (Fig. 4D). Consistently with previous results, the $\triangle \mathrm{TBP} 7$ mutant increased apoptotic cell death in scramble cells upon paclitaxel (Fig. 4C) and doxorubicin and epirubicin (Fig. 4D) treatment, as well as re-established drug sensitivity in docetaxel- (Fig. 4C) and epirubicin- and doxorubicin-resistant (Fig. 4D) cell models. According to our previous observations, these results are the consequence of the disabling of this cytoprotective pathway due to the disruption of TRAP1/TBP7 interaction upon transfection with the TBP7 deletion mutant (9).

The modulation of PERK phosphorylation by TRAPl correlates with resistance to anthracyclins. A major cytoprotective signal elicited by ER stress conditions is the phosphorylation of PERK which is responsible for the ATF4-dependent preferential translation of genes involved in cell metabolism and cytoprotective functions (31) and, among other, Bip/Grp78 (32). Since PERK upregulation in tumor cells has been recently proposed to induce the resistance to doxorubicin (33), we questioned whether the cytoprotective function of TRAP1 toward anthracyclins may involve the modulation of PERK pathway. In preliminary experiments, we observed the inhibition of PERK phosphorylation in MCF7 cells upon impairment of TRAP1/TBP7 pathway by the transfection of the $\triangle$ TBP7 deletion mutant (Fig. 5A). Since a reduced 


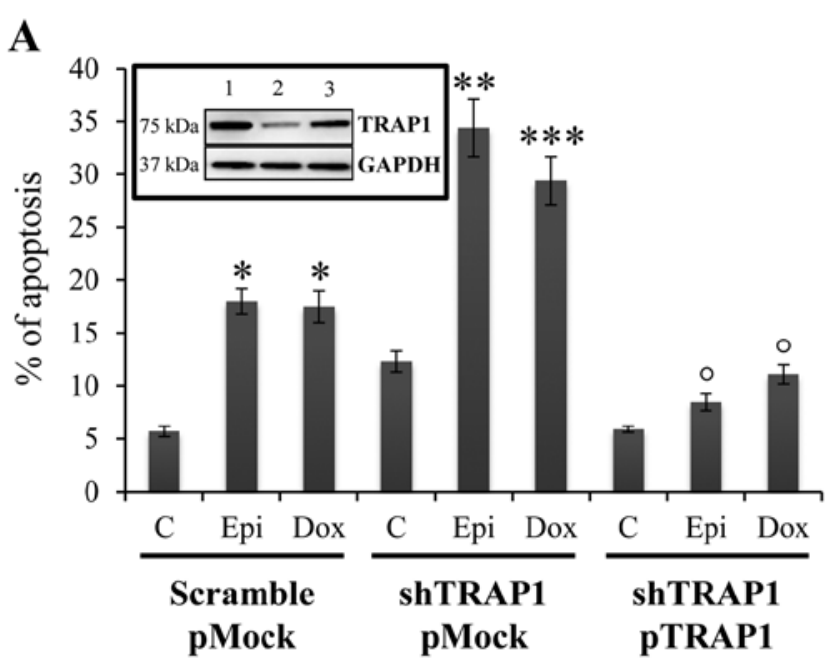

B

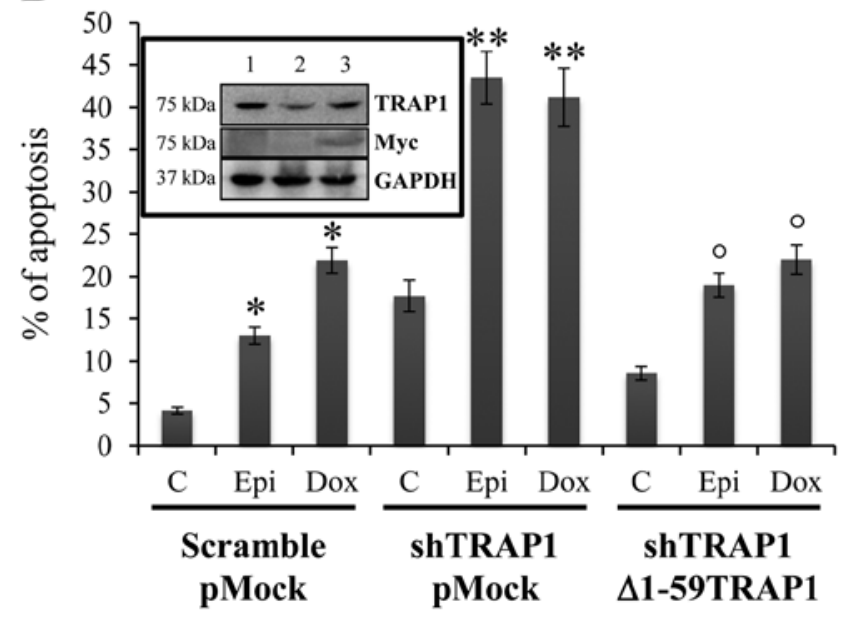

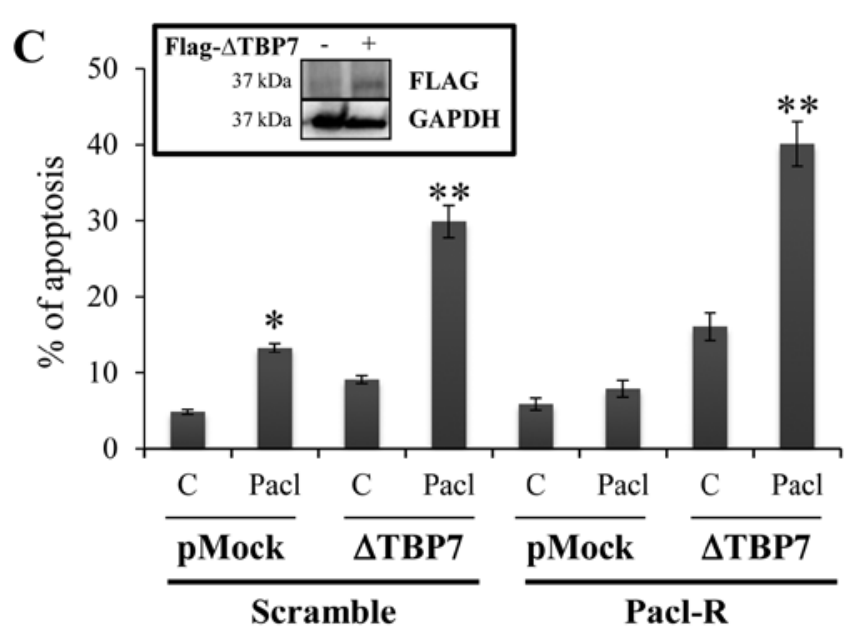

D

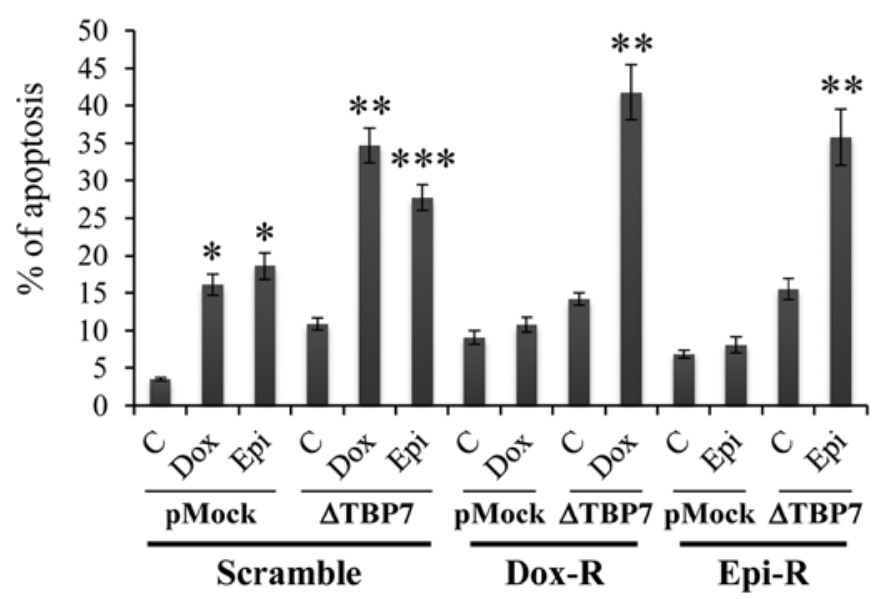

Figure 4. ER-associated TRAP1 favors resistance to anthracyclins. (A and B) Apoptotic levels in scramble, shTRAP1 MCF7 cells and shTRAP1 MCF7 cells transfected with TRAP1 cDNA (A) or the $\Delta 1-59 T R A P 1$ deletion mutant (B) and treated with $0.5 \mu \mathrm{M}$ epirubicin (Epi) or doxorubicin (Dox) for 24 h. P-values versus the respective untreated control $\left({ }^{*} \mathrm{p}<0.0001\right)$ or versus scramble cells transfected with pMock and treated with the same agent $\left({ }^{* *} \mathrm{p}<0.0001 ;{ }^{* * *} \mathrm{p}=0.002\right)$ or versus shTRAP1 cells transfected with pMock and treated with the same agent $\left({ }^{\circ} \mathrm{p}<0.0001\right)$. Inserts, TRAP1 levels in scramble, shTRAP1 MCF7 cells and shTRAP1 MCF7 cells transfected with TRAP1 cDNA (A) or the $\Delta 1-59 T R A P 1-M y c$ deletion mutant (B). (C) Apoptotic levels in scramble and paclitaxel-resistant (Pacl-R) MCF7 cells transfected with pMock or the $\Delta$ TBP7 deletion mutant treated with $1 \mu \mathrm{M}$ paclitaxel (Pacl) for $24 \mathrm{~h}$. Insert: $\Delta$ TBP7 mutant levels in MCF7 cells transfected with pMock or the Flag- $\Delta$ TBP7 deletion mutant. (D) Apoptotic levels in scramble, doxorubicin-resistant (Dox-R) and epirubicin-resistant (Epi-R) MCF7 cells transfected with pMock or the $\triangle \mathrm{TBP} 7$ deletion mutant treated with $0.5 \mu \mathrm{M}$ epirubicin (Epi) or doxorubicin (Dox) for $24 \mathrm{~h}$. (C and D) P-values versus the respective untreated control $\left({ }^{*} \mathrm{p}<0.0001\right)$ or versus pMock transfected cells treated with the same agent $\left({ }^{* *} \mathrm{p}<0.0001 ;{ }^{* * * *} \mathrm{p}=0.003\right)$.

phosphorylation of PERK was also observed in TRAP1 KD MCF7 cells compared to the scramble counterpart (Fig. 5B), these cell lines were exposed to a short-term treatment with doxorubicin and epirubicin. Of note, shTRAP1 cells failed to sustain PERK phosphorylation in response to anthracyclins (Fig. 5B), suggesting that the low expression of TRAP1 is likely responsible for the inability to elicit a cytoprotective response upon DNA damaging agents.

The downregulation of TRAPI and the simultaneous induction of ER stress by pharmacological agents result in additive apoptotic effects. Since our data suggest that TRAP1 protects from ER stress and that this function is relevant to elicit an antiapoptotic response upon ER stress conditions, we questioned whether the inhibition of TRAP1 may be clinically relevant in combination with pharmacological ER stress inducers (i.e., bortezomib and Mg132). The inhibition of TRAP1 was achieved by molecular strategies (i.e., transient downregulation by siRNA), this resulting by itself in reduced cell viability in MCF7 cells (Fig. 6A). Furthermore, the simultaneous inhibition of the proteasome activity with subcytotoxic concentrations of bortezomib and $\mathrm{Mg} 132$ resulted in a significant increase of apoptotic cell death (Fig. 6A). In agreement with our previous results, a lower activation of PERK phosphorylation in TRAP1-interefered cells compared to scramble cells is observed with the inability to sustain PERK phoshorylation in response to proteasome inhibitors (Fig. 6B). These results suggest that strategies that combine TRAP1 inhibition with agents that induce ER stress may achieve clinically-relevant synergistic/additive activities. 
A

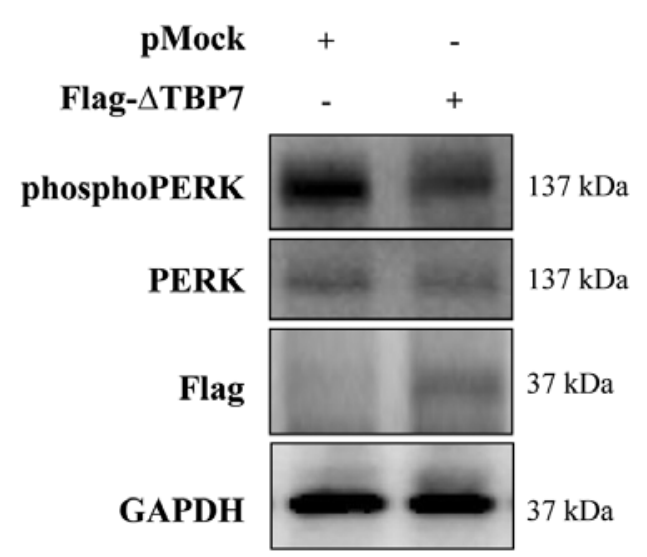

B

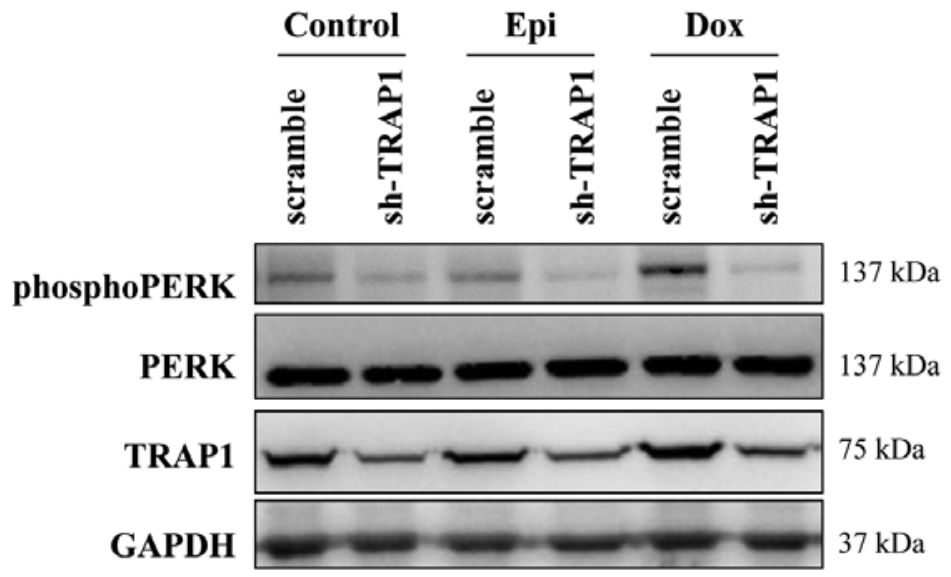

Figure 5. Modulation of PERK pathway by TRAP1 is responsible for resistance to anthracyclins. (A and B) Total cell lysates from MCF7 cells transfected with pMock or Flag- $\triangle \mathrm{TBP} 7$ mutant (A) or from scramble and shTRAP1 MCF7 treated with in $1 \mu \mathrm{M}$ epirubicin (Epi) or $5 \mu \mathrm{M}$ doxorubicin (Dox) for 10 min (B) were separated by SDS-PAGE and immunoblotted with anti-phosphoPERK, anti-PERK, anti-Flag and anti-GAPDH antibodies.

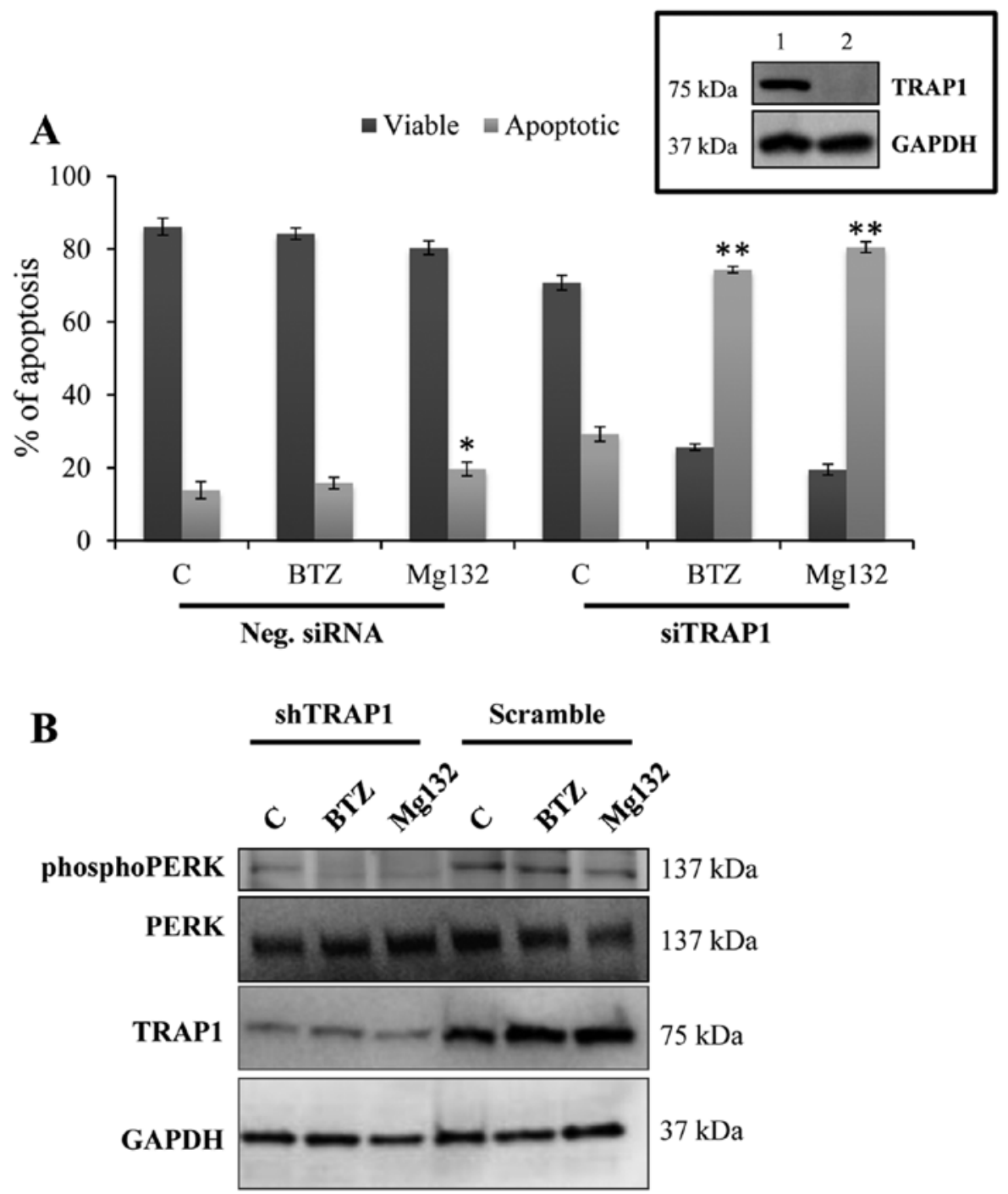

Figure 6. The cytotoxic activity of proteasome inhibitors is enhanced by TRAP1 downregulation. (A) Apoptotic levels in MCF7 cells transfected with control and TRAP1 siRNA and treated with $100 \mathrm{nM}$ bortezomib (BTZ) or $100 \mathrm{nM} \mathrm{Mg} 132$ for $12 \mathrm{~h}$. P-values versus the respective untreated control (" $\mathrm{p}=0.027$ ) or versus negative (Neg) siRNA transfected cells treated with the same agent ( $(* \mathrm{p}<0.0001)$. (B) Total cell lysates from scramble and shTRAP1 MCF7 treated with $100 \mathrm{nM}$ bortezomib (BTZ) or $100 \mathrm{nM} \mathrm{Mg132}$ for $6 \mathrm{~h}$ were separated by SDS-PAGE and immunoblotted with anti-phosphoPERK, anti-PERK, anti-TRAP1 and anti-GAPDH antibodies. 


\section{Discussion}

Cancer cells are exposed to chronic ER stress conditions and are, thus, characterized by the persistent activation of the ER UPR, with constitutive upregulation of several molecular chaperones and other cytoprotective genes, as well as activation of stress-related pathways. While excessive or prolonged UPR results in apoptosis $(2,3)$, mild chronic ER stress conditions are beneficial for cancer cells, favoring the adaptation to unfavorable environments and being responsible for antiapoptotic responses which ultimately lead to drug resistance (7). In such a context, recent evidence suggests that the ER UPR is responsible for inducing resistance to genotoxic agents and, among others, topoisomerase inhibitors (8). Indeed, the upregulation of the ER chaperone BiP/Grp78 protects against the cytotoxic activity of doxorubicin and cisplatin in human melanoma and gastric cancer cells $(8,21)$. Furthermore, genotoxic agents, besides their well known DNA damaging activity, favor the activation/upregulation of stress-related pathways that are responsible for cytoprotective responses (32). However, the mechanistic link between the ER stress response and prevention of apoptosis induced by DNA damaging agents is still poorly understood.

A putative role of TRAP1 in the ER stress control was initially suggested by Takemoto et al (10) who demonstrated that mitochondrial TRAP1 is associated with the activation of the UPR (10). Immediately after, this novel TRAP1 function was documented at molecular level showing that the selective targeting of HSP90 chaperones in mitochondria of human tumor cells triggers an organelle UPR and compensatory autophagy and that disabling this general adaptive pathway could potentially be used in the treatment of human tumors (11). In parallel studies, our group demonstrated that TRAP1, originally described as a mitochondrial protein, is also localized at the interface between ER and mitochondria where it is involved in crosstalk between these organelles, in this subcellular location TRAP1 interacts with TBP7, and plays a role in protection from ER stress, through a co-traslational regulation of protein ubiquitination and a quality control on specific mitochondrial client proteins $(9,34)$. Furthermore, this ER stress protecting activity by TRAP1 is crucial for its anti-apoptotic function: indeed, the quality control on specific mitochondrial proteins and, among others, $18 \mathrm{kDa}$ sorcin represents an additional mechanism used by this HSP90 chaperone to prevent apoptosis through the mitochondrial pathway (18). Based on the evidence that TRAP1 regulates the ER stress response and represents a key player in coupling organelle proteostasis and cell survival, through the regulation of the mitochondrial apoptotic pathway $(9-11,18)$, we explored its role in favoring resistance to anthracyclins in $\mathrm{BC}$ cells.

The present study demonstrated that i) the ER stress response is responsible for inducing resistance to epirubicin and doxorubicin; ii) ER-associated TRAP1 plays a key role in mediating this adaptive response to anthracyclins; and iii) this process is likely mediated by TRAP1 regulation of PERK pathway. In support of these conclusions, our data showed a cross-resistance between bortezomib and anthracyclins in both ER stress- and anthracyclin-adapted BC cell models and a regulatory function by TRAP1 in this process.
Of note, TRAP1 was upregulated upon short-term exposure to anthracyclins and in chronically-adapted drug-resistant cell lines and its silencing favored the re-establishment of drug sensitivity.

A major observation is the evidence that this cytoprotective response toward anthracyclins is driven by ER-associated TRAP1 and its ability to prevent ER stress. The evidence obtained by transfecting the TRAP1 mutant defective for mitochondrial import in TRAP1 KD cells and the TBP7 dominant negative deletion mutant in drug-resistant cell lines supports the notion that the ER stress protective function of TRAP1 is critical in favoring resistance to anthracyclins. Furthermore, our data suggest that this cytoprotective activity of TRAP1 likely relies on the modulation of PERK pathway. Indeed, PERK is an important stress sensor of the UPR, responsible for mediating phosphorylation of the $\alpha$ subunit of the eukaryotic translation initiation factor eIF2 (31), this leading in turn to global inhibition of protein synthesis and parallel ATF4-dependent preferential translation of genes involved in cell metabolism and cytoprotective functions (31). In this scenario, previous studies suggested that PERK represents a nodal point between ER stress and cell response to chemotherapeutics, being responsible for resistance to doxorubicin, through the interaction with the ER luminal protein ERp29 (33). Our study suggests that TRAP1 modulates the activity of PERK pathway and that this regulation is likely crucial for protection from cytotoxics. In such a scenario, high TRAP1 levels in tumor cells may represent a prerequisite for enabling an efficient cytoprotective ER stress response and this may represent a major mechanism responsible for drug resistance. Specifically in this report, this function is linked, for the first time, to our knowledge, to protection toward anthracyclins, agents that primarily act by inhibiting topisomerase and inducing DNA damage. Interestingly, previous studies by our group have shown TRAP1 involvent in the protection against taxanes (18), agents that disregulate protein homeostasis $(35,36)$. In this perspective, our studies highlight the relevance of the ER stress protective activity of TRAP1, as complementary/additive respect to its well known antiapoptotic mitochondrial function (36), in favoring resistance to apoptosis through the regulation of protein quality and the modulation of mitochondrial apoptotic pathway. Thus, TRAP1 targeting in the ER may be regarded as a strategy to disable the crosstalk between ER and mitochondria and, eventually, revert resistance to cytotoxics.

Novel HSP90/TRAP1 inhibitors are under preclinical evaluation, as valuable anticancer agents to be used as single agents or in combination with other anticancer drugs in the treatment of human malignancies (37). We observed that the simultaneous targeting of the ER cytoprotective pathway by the downregulation of TRAP1, which is a much more specific approach compared to chemical ER stress inducers, and the parallel inhibition of the proteasome activity results in a dramatic increase in apoptotic cell death. This additive/synergistic activity between TRAP1 silencing and stress agents represents the proof of principle that the disabling of cytoprotective responses upon TRAP1 suppression, and, among others, PERK phosphorylation, may enhance the cytotoxic activity of agents that target the ER response (i.e., ER stress agents, taxanes, anthracyclins). Thus, TRAP1 inhibition may 
represent a novel strategy to selectively block relevant cytoprotective pathways used by cancer cells to escape apoptosis and acquire a drug resistant phenotypes. These observation may be clinically relevant in human $\mathrm{BCs}$ that are characterized by the co-upregulation of TRAP1 and BiP/Grp78 in about $50 \%$ of cases and likely use these adaptive responses to acquire resistance to apoptosis (18). Furthermore, specific BC subtypes, i.e. HER2-positive or triple negative breast cancers, may represent ideal human tumor models to evaluate antiHSP90 chaperone therapy, based on the evidence that HER2 in a well known HSP90 client protein $(38,39)$, whereas triple negative BCs are aggressive drug-resistant malignancies which lack specific molecular targets for treatment (40). In such a perspective, our data provide the preclinical rationale for evaluating TRAP1/HSP90 targeting as a strategy to revert resistance to apoptosis in selected human malignancies.

\section{Acknowledgements}

This study was supported by grants from the Associazione Italiana per la Ricerca sul Cancro (AIRC, IG13128) to M.L. and F.E., the Italian Ministry of Health (GR-2010-2310057 E66I10000220001) to F.M. and POR Campania FSE 2007-2013, project crème to F.E.

\section{References}

1. Walter P and Ron D: The unfolded protein response: from stress pathway to homeostatic regulation. Science 334: 1081-1086, 2011.

2. Schröder M and Kaufman RJ: The mammalian unfolded protein response. Annu Rev Biochem 74: 739-789, 2005.

3. Ron D and Walter P: Signal integration in the endoplasmic reticulum unfolded protein response. Nat Rev Mol Cell Biol 8: 519-529, 2007.

4. Ellgaard L and Helenius A: Quality control in the endoplasmic reticulum. Nat Rev Mol Cell Biol 4: 181-191, 2003.

5. Ron D: Translational control in the endoplasmic reticulum stress response. J Clin Invest 110: 1383-1388, 2002.

6. Tassone P, Tagliaferri P, Fulciniti MT, Di Martino MT and Venuta S: Novel therapeutic approaches based on the targeting of microenvironment-derived survival pathways in human cancer: experimental models and translational issues. Curr Pharm Des 13: 487-496, 2007.

7. Wilson TR, Johnston PG and Longley DB: Anti-apoptotic mechanisms of drug resistance in cancer. Curr Cancer Drug Targets 9: 307-319, 2009

8. Reddy RK, Mao C, Baumeister P, Austin RC, Kaufman RJ and Lee AS: Endoplasmic reticulum chaperone protein GRP78 protects cells from apoptosis induced by topoisomerase inhibitors: role of ATP binding site in suppression of caspase-7 activation. J Biol Chem 278: 20915-20924, 2003.

9. Amoroso MR, Matassa DS, Laudiero G, et al: TRAP1 and the proteasome regulatory particle TBP7/Rpt3 interact in the endoplasmic reticulum and control cellular ubiquitination of specific mitochondrial proteins. Cell Death Differ 19: 592-604, 2012.

10. Takemoto $\mathrm{K}$, Miyata S, Takamura $\mathrm{H}$, Katayama $\mathrm{T}$ and Tohyama M: Mitochondrial TRAP1 regulates the unfolded protein response in the endoplasmic reticulum. Neurochem Int 58: 880-887, 2011.

11. Siegelin MD, Dohi T, Raskett CM, et al: Exploiting the mitochondrial unfolded protein response for cancer therapy in mice and human cells. J Clin Invest 121: 1349-1360, 2011

12. Kang BH, Plescia J, Dohi T, Rosa J, Doxsey SJ and Altieri DC: Regulation of tumor cell mitochondrial homeostasis by an organelle-specific Hsp90 chaperone network. Cell 131: 257-270, 2007.

13. Costantino E, Maddalena F, Calise S, et al: TRAP1, a novel mitochondrial chaperone responsible for multi-drug resistance and protection from apoptotis in human colorectal carcinoma cells. Cancer Lett 279: 39-46, 2009.
14. Leav I, Plescia J, Goel HL, et al: Cytoprotective mitochondrial chaperone TRAP-1 as a novel molecular target in localized and metastatic prostate cancer. Am J Pathol 176: 393-340, 2009.

15. Fang W, Li X, Jiang Q, et al: Transcriptional patterns, biomarkers and pathways characterizing nasopharyngeal carcinoma of Southern China. J Transl Med 6: 32, 2008.

16. Landriscina M, Maddalena F, Laudiero G and Esposito F: Adaptation to oxidative stress, chemoresistance, and cell survival. Antioxid Redox Signal 11: 2701-2716, 2009.

17. Landriscina M, Laudiero G, Maddalena F, et al: Mitochondrial chaperone Trap1 and the calcium binding protein Sorcin interact and protect cells against apoptosis induced by antiblastic agents. Cancer Res 70: 6577-6586, 2010.

18. Maddalena F, Sisinni L, Lettini G, et al: Resistance to paclitxel in breast carcinoma cells requires a quality control of mitochondrial antiapoptotic proteins by TRAP1. Mol Oncol 7: 895-906, 2013.

19. Hande KR: Clinical applications of anticancer drugs targeted to topoisomerase II. Biochim Biophys Acta 1400: 173-184, 1998.

20. Minotti G, Menna P, Salvatorelli E, Cairo G and Gianni L: Anthracyclines: molecular advances and pharmacologic developments in antitumor activity and cardiotoxicity. Pharmacol Rev 56: 185-229, 2004

21. Jiang CC, Mao ZG, Avery-Kiejda KA, Wade M, Hersey P and Zhang XD: Glucose-regulated protein 78 antagonizes cisplatin and adriamycin in human melanoma cells. Carcinogenesis 30: 197-204, 2009.

22. Chae YC, Caino MC, Lisanti S, et al: Control of tumor bioenergetics and survival stress signaling by mitochondrial HSP90s. Cancer Cell 22: 331-344, 2012.

23. Scriven P, Coulson S, Haines R, Balasubramanian S, Cross S and Wyld L: Activation and clinical significance of the unfolded protein response in breast cancer. Br J Cancer 101: 1692-1698, 2009.

24. Tan SS, Ahmad I, Bennett HL, et al: GRP78 up-regulation is associated with androgen receptor status, Hsp70-Hsp90 client proteins and castrate-resistant prostate cancer. J Pathol 223: 81-87, 2011

25. Khasraw M, Bell R and Dang C: Epirubicin: is it like doxorubicin in breast cancer? A clinical review. Breast 21: 142-149, 2012

26. Barone C,Landriscina M, Quirino M, et al: Schedule-dependent activity of 5-fluorouracil and irinotecan combination in the treatment of human colorectal cancer: in vitro evidence and a phase I dose-escalating clinical trial. Br J Cancer 96: 21-28, 2007.

27. Landriscina M, Fabiano A, Altamura S, et al: Reverse transcriptase inhibitors down-regulate cell proliferation in vitro and in vivo and restore thyrotropin signaling and iodine uptake in human thyroid anaplastic carcinoma. J Clin Endocrinol Metab 90: 5663-5671, 2005.

28. Maddalena F, Laudiero G, Piscazzi A, et al: Sorcin induces a drug-resistant phenotype in human colorectal cancer by modulating $\mathrm{Ca}(2+)$ homeostasis. Cancer Res 71: 7659-7669, 2011.

29. Feng R, Zhai WL, Yang HY, Jin H and Zhang QX: Induction of ER stress protects gastric cancer cells against apoptosis induced by cisplatin and doxorubicin through activation of p38 MAPK. Biochem Biophys Res Commun 406: 299-304, 2011.

30. Mhaidat NM, Thorne R, Zhang XD and Hersey P: Involvement of endoplasmic reticulum stress in Docetaxel-induced JNK-dependent apoptosis of human melanoma. Apoptosis 13: 1505-1512, 2008.

31. Harding HP, Zhang Y and Ron D: Protein translation and folding are coupled by an endoplasmic-reticulum-resident kinase. Nature 397: 271-274, 1999.

32. Harding HP, Novoa I, Zhang Y, et al: Regulated translation initiation controls stress-induced gene expression in mammalian cells. Mol Cell 6: 1099-1108, 2000.

33. Farmaki E, Mkrtchian S, Papazian I, Papavassiliou AG and Kiaris H: ERp29 regulates response to doxorubicin by a PERK-mediated mechanism. Biochim Biophys Acta 1813: 1165-1171, 2011.

34. Matassa DS, Amoroso MR, Crudele V, et al: Translational control in the stress adaptive response of cancer cells: a novel role for the heat shock protein TRAP1. Cell Death Dis 4: e851, doi: 10.1038/ cddis.2013.379, 2013.

35. Wang J, Yin Y, Hua $\mathrm{H}$ et al: Blockade of GRP78 sensitizes breast cancer cells to microtubules-interfering agents that induce the unfolded protein response. J Cell Mol Med 13: 3888-3897, 2009. 
36. Kang BH: TRAP1 regulation of mitochondrial life or death decision in cancer cells and mitochondria-targeted TRAP1 inhibitors. BMB Rep 45: 1-6, 2012.

37. Neckers L, Kern A and Tsutsumi S: Hsp90 inhibitors disrupt mitochondrial homeostasis in cancer cells. Chem Biol 14: 1204-1206, 2007.

38. Xu W, Mimnaugh E, Rosser MF et al: Sensitivity of mature Erbb2 to geldanamycin is conferred by its kinase domain and is mediated by the chaperone protein Hsp90. J Biol Chem 276: 3702-3708, 2001.
39. Basso AD, Solit DB, Munster PN and Rosen N: Ansamycin antibiotics inhibit Akt activation and cyclin D expression in breast cancer cells that overexpress HER2. Oncogene 21: 1159-1166, 2002.

40. Brouckaert O, Wildiers H, Floris G and Neven P: Update on triple-negative breast cancer: prognosis and management strategies. Int J Womens Health 4: 511-520, 2012. 